\title{
Quasi-isometrically embedded free sub-semigroups
}

\author{
YVES DE CORNULIER \\ ROMAIN TESSERA
}

\begin{abstract}
If $G$ is either a connected Lie group, or a finitely generated solvable group with exponential growth, we show that $G$ contains a quasi-isometrically embedded free sub-semigroup on 2 generators.
\end{abstract}

20F65; 20F16, 20M05, 22E25, 20E05

\section{Introduction}

Let $G$ be a locally compact group generated by a compact subset $S$. The word length function is defined in the usual way: $|g|_{S} \leq n$ if and only if $g$ is the product of $n$ elements of $S^{ \pm 1}$. If $G$ is a connected Lie group, then it can also be endowed with the distance defined by a left invariant Riemannian metric. All corresponding lengths are equivalent, where two lengths $\ell, \ell^{\prime}$ are called equivalent if $\ell \preceq \ell^{\prime} \preceq \ell$ and $\ell \preceq \ell^{\prime}$ means that there exists a constant $\alpha>0$ such that for all $g$ in the group, $\ell(g) \leq \alpha \ell^{\prime}(g)+\alpha$. In the sequel, compactly generated groups will be endowed with a length equivalent to one of these, the choice of which has no importance.

We are especially interested in quasi-isometric homomorphic embeddings of a free semigroup into some compactly generated groups.

Recall that a map $f: X \rightarrow Y$ between two metric spaces is large scale Lipschitz if

$$
\exists \alpha>0 \text { such that } \forall x, y \in X, d(f(x), f(y)) \leq \alpha d(x, y)+\alpha .
$$

It is called a quasi-isometric embedding if moreover it satisfies

$$
\exists \beta>0 \text { such that } \forall x, y \in X, \beta d(x, y)-\beta \leq d(f(x), f(y)) .
$$

In the sequel, $X=\Gamma$ will mainly denote a free (non-abelian) semigroup on two generators, which we simply call "the" free semigroup, in which case it is endowed with the metric of the Cayley graph with respect to the given free generators.

It is then straightforward that any homomorphism $f$ of $\Gamma$ into a compactly generated group is large scale Lipschitz. On the other hand, $f$ is not necessarily a quasi-isometric 
embedding: for instance this is obvious when $f$ is not injective. Also, there are many proper homomorphisms of free (semi)groups that are not quasi-isometric embeddings, eg the embedding of a finite index free subgroup of $\mathrm{SL}_{2}(\mathbf{Z})$ into $\mathrm{SL}_{2}(\mathbf{R})$ (or into $\left.\mathrm{SL}_{2}(\mathbf{Z}[1 / 2])\right)$. The lack of being a quasi isometric embedding can be encoded in the compression function

$$
\rho(r)=\inf \{d(f(x), f(y)): x, y \in X, d(x, y) \geq r\} .
$$

Then the large-scale Lipschitz map $f$ is a quasi-isometric embedding if and only if $\rho(r) \succeq r$ (or equivalently $\rho(r) \simeq r$ if $X$ is unbounded). There is much work concerning the compression function (and also the related distortion function), see for instance Gromov [7], Farb [6], Ol'shanskiǔ [9] and Osin [11].

Our interest for the existence of quasi-isometrically embedded free subsemigroups comes from the following result of Bourgain [4]: a regular tree of degree at least three has no quasi-isometric embedding into a Hilbert space (nor into any uniformly convex Banach space); note that a free semigroup is quasi-isometric to such a tree.

Existence of a quasi-isometrically embedded free subsemigroup is also a strengthening of the existence of a free subsemigroup. We prove for instance the following theorem, which improves the Rosenblatt alternative [12].

Theorem 1.1 Let $\Gamma$ be a finitely generated solvable group. Suppose that $\Gamma$ is not virtually nilpotent. Then $\Gamma$ has a quasi-isometrically embedded free subsemigroup.

We give two related results for non-discrete groups.

Theorem 1.2 Let $G$ be a compactly generated group that is not of polynomial growth. Suppose that either

(1) $G$ is a connected Lie group, or

(2) $G$ is a linear algebraic group over a local field of characteristic zero.

Then $G$ has a quasi-isometrically embedded free semigroup.

This theorem is not true for general compactly generated groups, eg the free Burnside groups of odd large exponent, which have exponential growth (Adian [1]) but do not even contain any homomorphically embedded copy of $\mathbf{Z}$. On the other hand, it is not known if every locally compact compactly generated group with exponential growth $G$ contains a quasi-isometrically embedded regular ternary tree. This is known to hold for non-amenable $G$ and non-unimodular $G$ : more precisely, it follows from the two following facts. On the one hand, it is known [2, Corollary 1.6] that the regular ternary 
tree quasi-isometrically embeds into every graph with positive Cheeger constant. On the other hand, by Tessera [14, Theorem 2], if $G$ is non-amenable or non-unimodular, then $G$ is quasi-isometric to a graph with positive Cheeger constant.

From our results we get, relying on Bourgain [4], the following corollary.

Corollary 1.3 Let $G$ be given as in Theorem 1.1 or 1.2. Then $G$ has a quasiisometrically embedded tree, and in particular $G$ does not quasi-isometrically embed into any uniformly convex (or superreflexive) Banach space.

Further consequences on the compression of such an embedding can be found in Tessera [13].

The usual ping-pong Lemma gives a combinatorial condition ensuring that two transformations of a set generate a free subsemigroup. To obtain quasi-isometrically embedded semi-groups, we formulate in Section 2 a "quasi-isometric ping-pong Lemma", which holds for isometric group actions on metric spaces satisfying a suitable geodesic condition. The use of this ping-pong Lemma in the hyperbolic space, showing for instance that two hyperbolic transformations with distinct targets generate a free subsemigroup, is known as the "Schottky argument"; in that case it is indeed possible to apply the quasi-isometric ping-pong Lemma. This works for instance inside every Zariski dense subgroup of $\mathbf{K}^{*} \ltimes \mathbf{K}$, where $\mathbf{K}$ is a local field. This group acts properly cocompactly on the two or three-dimensional real hyperbolic space if $\mathbf{K}$ is Archimedean, or on a locally finite tree when $\mathbf{K}$ is ultrametric. Groves [8] proved that every finitely generated non-virtually nilpotent group has a finite index subgroup having a homomorphism with Zariski dense image into $\mathbf{K}^{*} \ltimes \mathbf{K}$ for some local field $\mathbf{K}$. The quasi-isometric pingpong Lemma thus allows to find a quasi-isometrically embedded free subsemigroup. Groves's result allowing, starting from any finitely generated solvable group, to play ping-pong in $\mathbf{K}^{*} \ltimes \mathbf{K}$ is also used by E Breuillard in [5].

Finally, let us notice that there exists finitely generated groups $G$ containing a nonabelian free semigroup (actually a non-abelian free group), but which cannot be chosen quasi-isometrically embedded. Indeed, Osin [10] has proved that any countable torsionfree group $H$ (eg a non-abelian free group) can be embedded into a 2-generated group $G$ in which all non-trivial elements are conjugate. Plainly, $G$ is torsion-free. Moreover, as every element is conjugate to its square, every element is at least exponentially distorted (ie for every $x$, the word length of $x^{n}$ grows at most like $\log (n)$ ). Thus $G$ contains no quasi-isometrically embedded semigroup on one generator, and therefore neither on two generators.

Acknowledgments We thank the referee for useful remarks, and D Osin for pointing out his paper [10]. 


\section{The quasi-isometric ping-pong Lemma}

We call here a metric space 1-quasi-geodesic if there exists a constant $c$ such that every two points $x, y$ are joined by a "path" $x=x_{0}, \ldots, x_{n}=y$ with $n \leq c d(x, y)+c$ and $d\left(x_{i}, x_{i+1}\right) \leq 1$ for all $i$.

In any metric space $X$, denote by $B_{r}(x)$ the closed ball of centre $x$ and radius $r$, and, for $Y \subset X$, define

$$
\mathcal{B}_{r}(Y)=\bigcup_{y \in Y} B_{r}(y)
$$

Lemma 2.1 (Quasi-isometric ping-pong Lemma for semigroups) Let $X$ be a 1quasi-geodesic space and let $\left(a_{i}\right)_{i \in I}, I=\{1 \ldots d\}(d \geq 2)$ be a family of surjective isometries of $X$. Assume that there exists a family of nonempty disjoint subsets $\left(A_{i}\right)_{i \in I}$ such that, setting $C=\bigcup_{i} A_{i}$,

$$
\forall i \in I, \quad \mathcal{B}_{1}\left(a_{i} C\right) \subset A_{i} .
$$

Then, for every $x \in X$, the mapping $m \mapsto m\left(a_{1}, \ldots, a_{d}\right) x$ defines a quasi-isometric embedding of the free semigroup of rank $d$ into $X$.

In particular, if $G$ is a locally compact compactly generated group, with elements $x_{1}, \ldots, x_{d}$ and if $G$ acts by isometries on $X$ in such a way that $x_{i}$ acts by $a_{i}$, then $x_{1}, \ldots, x_{d}$ freely generate a quasi-isometrically embedded free subsemigroup of $G$.

Given a metric space $X$, view $\mathcal{B}_{1}$ as a self-map of $2^{X}$, and denote by $\mathcal{B}_{1}^{n}$ its $n$-th iterate. The triangle inequality yields, for all $Y \subset X$ and $n \geq 0$ the inclusion $\mathcal{B}_{1}^{n}(Y) \subset \mathcal{B}_{n}(Y)$.

Lemma 2.2 A metric space $X$ is 1-quasi-geodesic if and only if there exists a constant $c>0$ such that for all $Y \subset X$ and $n \in \mathbf{N}^{*}$, we have

$$
\mathcal{B}_{1}^{n}(Y) \supset \mathcal{B}_{c n}(Y)
$$

Proof Suppose that $X$ is quasi-geodesic, so that there exist constants $c_{1} \geq 1, c_{2} \geq 0$ such that for every two points $x, y \in X$ there exist $x=x_{0}, \ldots, x_{n}=y$ with $n \leq$ $c_{1} d(x, y)+c_{2}$ and $d\left(x_{i}, x_{i+1}\right) \leq 1$ for all $i$. Set $c=\left(c_{1}+c_{2}\right)^{-1}$. We claim that for all $Y \subset X$ and $n \in \mathbf{N}^{*}$, we have $\mathcal{B}_{1}^{n}(Y) \supset \mathcal{B}_{c n}(Y)$. Indeed, take $n \in \mathbf{N}^{*}$. If $n \leq c_{1}+c_{2}$, then $c n \leq 1$, so that $\mathcal{B}_{c n}(Y) \subset \mathcal{B}_{1}(Y) \subset \mathcal{B}_{1}^{n}(Y)$. Otherwise $n>c_{1}+c_{2}$. If $x \in \mathcal{B}_{c n}(Y)$, then by the 1-quasi-geodesic condition, $x \in \mathcal{B}_{1}^{m}(Y)$ for some integer $m \leq c_{1} c n+c_{2}$. On the other hand $n-c_{1} c n=n c_{2} /\left(c_{1}+c_{2}\right) \geq c_{2}$ as $n \geq c_{1}+c_{2}$. Therefore $m \leq n$, proving the implication. 
Conversely if the condition of the lemma is satisfied, then it follows that for all $x, y \in X$, there exist $x=x_{0}, \ldots, x_{n}=y$ satisfying $n \leq\lceil d(x, y) / c\rceil$ and $d\left(x_{i}, x_{i+1}\right) \leq 1$ for all $i$. Now $\lceil d(x, y) / c\rceil \leq c^{-1} d(x, y)+1$, so $X$ is 1 -quasi-geodesic.

\section{Proof of Lemma 2.1}

Claim 2.3 Let $m=a_{j_{1}} \ldots a_{j_{n}}$ be a word of length $n \geq 1$ in $\left(a_{i}\right)_{i \in I}$. Then

$$
\mathcal{B}_{1}^{n}(m(C)) \subset A_{j_{1}} \text {. }
$$

Proof First note that since every $a_{j}$ is a surjective isometry, it commutes with the map $\mathcal{B}_{1}$ and its iterates. Argue by induction on $n$. For $n=1$, the claim is contained in the hypotheses of the theorem. Fix $n \geq 2$, and assume that the lemma holds for $n-1$. Write $m^{\prime}=a_{j_{2}} \ldots a_{j_{n}}$. We have

$$
\begin{aligned}
\mathcal{B}_{1}^{n}(m(C)) & =\mathcal{B}_{1} \circ \mathcal{B}_{1}^{n-1}\left(a_{j_{1}} m^{\prime}(C)\right) \\
& =\mathcal{B}_{1}\left(a_{j} \mathcal{B}_{1}^{n-1}\left(m^{\prime}(C)\right)\right) \\
& \subset \mathcal{B}_{1}\left(a_{j_{1}} A_{j_{2}}\right) \text { (by induction assumption) } \\
& \subset A_{j_{1}}
\end{aligned}
$$

This completes the proof.

Denote by $\delta$ the word distance in the free semigroup generated by the letters $\left(a_{i}\right)_{i \in I}$.

Consider two words $m, m^{\prime}$. Denoting by $v$ their greatest common prefix, we can write $m=v a_{i_{1}} \ldots a_{i_{n}}$ and $m^{\prime}=v a_{j_{1}} \ldots a_{j_{k}}$, where $n+k=\delta\left(m, m^{\prime}\right)$. If $k, n>0$ then the claim implies that

$$
\mathcal{B}_{1}^{n}\left(v^{-1} m(C)\right) \subset A_{i_{1}}, \quad \mathcal{B}_{1}^{k}\left(v^{-1} m^{\prime}(C)\right) \subset A_{j_{1}} .
$$

As $A_{i_{1}} \cap A_{j_{1}}=\varnothing$, we deduce that $\mathcal{B}_{1}^{n}\left(v^{-1} m(C)\right) \cap \mathcal{B}_{1}^{k}\left(v^{-1} m^{\prime}(C)\right)=\varnothing$ and therefore

$$
\mathcal{B}_{c n}\left(v^{-1} m(C)\right) \cap \mathcal{B}_{c k}\left(v^{-1} m^{\prime}(C)\right)=\varnothing .
$$

Now fix $x_{0}$ in $C$, which is not empty. From the previous formula we get

$$
d\left(m x_{0}, m^{\prime} x_{0}\right)=d\left(v^{-1} m x_{0}, v^{-1} m^{\prime} x_{0}\right) \geq c(n+k) .
$$

The case $k n=0$ remains. The case $k=n=0$ being obvious, we can suppose $k=0 \neq n$. Fix $j \neq i_{1}$. Then

$$
\begin{gathered}
d\left(m x_{0}, m^{\prime} x_{0}\right) \geq d\left(m x_{0}, v a_{j} x_{0}\right)-d\left(v a_{j} x_{0}, m^{\prime} x_{0}\right) \\
=d\left(m x_{0}, v a_{j} x_{0}\right)-d\left(a_{j} x_{0}, x_{0}\right) \geq c(k+n+1)-d\left(a_{j} x_{0}, x_{0}\right) \geq c \delta\left(m, m^{\prime}\right)-\alpha,
\end{gathered}
$$


where $\alpha=\sup _{j} d\left(a_{j} x_{0}, x_{0}\right)$.

We immediately deduce, for every $x \in X$ and all words $m, m^{\prime}$,

$$
d\left(m x, m^{\prime} x\right) \geq c \delta\left(m, m^{\prime}\right)-\alpha^{\prime},
$$

where $\alpha^{\prime}=\alpha+2 d\left(x, x_{0}\right)$.

Lemma 2.4 (Quasi-isometric ping-pong Lemma for groups) Let $X$ be a 1-quasigeodesic space and let $\left(a_{i}\right)_{i \in I}, I=\{1, \ldots d\}(d \geq 1)$ be a family of surjective isometries of $X$. Consider a family of $2 d$ disjoint nonempty subsets $\left(A_{i}^{ \pm}\right)_{i \in I}$. For every $i \in I$, write

$$
C_{i}^{+}=\left(\bigcup_{j \in I} A_{j}^{+}\right) \cup\left(\bigcup_{j \neq i} A_{j}^{-}\right) \text {and } C_{i}^{-}=\left(\bigcup_{j \in I} A_{j}^{-}\right) \cup\left(\bigcup_{j \neq i} A_{j}^{+}\right) .
$$

Assume that, for every $i \in I$,

$$
\mathcal{B}_{1}\left(a_{i}^{+}\left(C_{i}^{+}\right)\right) \subset A_{i}^{+} \text {and } \mathcal{B}_{1}\left(a_{i}^{-}\left(C_{i}^{-}\right)\right) \subset A_{i}^{-} .
$$

Then for every $x \in X$, the mapping $m \rightarrow m\left(a_{1}, \ldots, a_{d}\right) x$ defines a quasi-isometric embedding of the free group of rank $d$ into $X$.

In particular, if $G$ is a locally compact compactly generated group, with elements $x_{1}, \ldots, x_{d}$ and if $G$ acts by isometries on $X$ in such a way that $x_{i}$ acts by $a_{i}$, then $x_{1}, \ldots, x_{d}$ are free generators of a quasi-isometrically embedded free subgroup of $G$.

Proof The following claim is similar to Claim 2.3.

Claim 2.5 Let $m=a_{j_{1}}^{\varepsilon_{1}} \ldots a_{j_{n}}^{\varepsilon_{n}}$ be a reduced word of length $n \geq 1$ in $\left(a_{i}^{ \pm}\right)$, with $i \in\{1, \ldots, n\}, \varepsilon_{i} \in\{ \pm\}$. Then

$$
\mathcal{B}_{1}^{n}\left(m\left(C_{j_{n}}^{\varepsilon_{n}}\right)\right) \subset A_{j_{1}}^{\varepsilon_{1}}
$$

Proof Argue by induction on $n$, the case $n=1$ being obvious. If $n \geq 2$ and the claim is proved for $n-1$, then $\mathcal{B}_{1}^{n-1}\left(a_{j_{2}}^{\varepsilon_{1}} \ldots a_{j_{n}}^{\varepsilon_{n}} C_{j_{n}}^{\varepsilon_{n}}\right)$ is contained in $A_{j_{2}}^{\varepsilon_{2}}$, so that $\mathcal{B}_{1}^{n}\left(m\left(C_{j_{n}}^{\varepsilon_{n}}\right)\right)$ is contained in $\mathcal{B}_{1}\left(a_{j_{1}} A_{j_{2}}^{\varepsilon_{2}}\right)$, which is contained in $A_{j_{1}}^{\varepsilon_{1}}$, noting that $\left(j_{1}, \varepsilon_{1}\right) \neq\left(j_{2},-\varepsilon_{2}\right)$ as the word is reduced.

Denote by $\delta$ the word distance in the free group generated by the letters $\left(a_{i}\right)_{i \in I}$. For each $m$ as in the claim, we can find $x_{m} \in A_{j}^{\varepsilon}$ where $(j, \varepsilon) \notin\left\{\left(j_{1}, \varepsilon_{1}\right),\left(j_{n},-\varepsilon_{n}\right)\right\}$; the mapping $m \mapsto x_{m}$ can be chosen so that it has finite image (of cardinality 3 for instance). 
By construction $x_{m} \in C_{j_{n}}^{\varepsilon_{n}}-A_{j_{1}}^{\varepsilon_{1}}$. It follows from the claim that $\mathcal{B}_{c n}\left(m\left(C_{j_{n}}^{\varepsilon_{n}}\right)\right) \subset A_{j_{1}}^{\varepsilon_{1}}$, so that

$$
d\left(m x_{m}, x_{m}\right) \geq c n=c \delta(m, 1) .
$$

Accordingly, if $x \in X$, we have for every word $m$

$$
d(m x, x) \geq c \delta(m, 1)-2 \alpha,
$$

where $\alpha=\sup \left\{d\left(x_{m}, x\right): m\right.$ reduced word $\}<\infty$. This shows that the mapping $m \mapsto m x$ is a quasi-isometric embedding of the free group.

\section{Ping-pong in dimension two}

The following lemma is used both in the case of amenable Lie groups and of finitely generated virtually solvable groups.

Lemma 3.1 Let $\mathbf{K}$ be a local field. Consider the $(a x+b)$-group

$$
G=\mathbf{K} \rtimes \mathbf{K}^{*} \simeq\left\{\left(\begin{array}{ll}
a & b \\
0 & 1
\end{array}\right): a \in \mathbf{K}^{*}, b \in \mathbf{K}\right\} .
$$

Let

$$
A_{1}=\left(\begin{array}{cc}
a_{1} & b_{1} \\
0 & 1
\end{array}\right), \quad A_{2}=\left(\begin{array}{cc}
a_{2} & b_{2} \\
0 & 1
\end{array}\right)
$$

be non-commuting elements in $\mathbf{K} \rtimes \mathbf{K}^{*}$ and suppose that

- $\mathbf{K}$ is ultrametric and $\left|a_{1}\right|,\left|a_{2}\right|<1$, or

- $\mathbf{K}$ is Archimedean and $\left|a_{1}\right|+\left|a_{2}\right|+3\left|a_{1}\right|\left|a_{2}\right|<1$ (eg $\left.\left|a_{1}\right|,\left|a_{2}\right|<1 / 3\right)$.

Then the sub-semigroup generated by $A_{1}$ and $A_{2}$ is free and quasi-isometrically embedded in $\mathbf{K} \rtimes \mathbf{K}^{*}$.

Proof In the Archimedean case, we can suppose $\mathbf{K}=\mathbf{C}$. We make $G$ act in the standard way by isometries on the hyperbolic 3-space $\mathbf{H}_{\mathbf{R}}^{3}$, viewed in the half-space model $\mathbf{C} \times \mathbf{R}_{+}^{*}$, and on its boundary $\mathbf{P}^{1}(\mathbf{C})=\mathbf{C} \cup\{\infty\}$ as the stabilizer of $\infty$.

We are going to apply the quasi-isometric ping-pong lemma by choosing two halfspaces. Note that taking the boundary gives a natural correspondance between the set of all closed half-spaces in $\mathbf{H}_{\mathbf{R}}^{3}$ that do not contained $\infty$ in their closure, and the set of closed Euclidean discs (of bounded radius) in $\mathbf{C}$. 
For $i \in\{1,2\}, A_{i}$ is a hyperbolic element with repulsive point $\infty$ and attractive point $x_{i} \in \mathbf{C}$. Set $d=\left|x_{2}-x_{1}\right|$. As $A_{1}$ and $A_{2}$ do not commute by assumption, we have $d>0$. If $\{1,2\}=\{i, j\}$, set

$$
r_{i}=\frac{d\left|a_{i}\right|\left(1+\left|a_{j}\right|\right)}{1-\left|a_{1}\right|\left|a_{2}\right|} .
$$

The assumption $\left|a_{1}\right|+\left|a_{2}\right|+3\left|a_{1}\right|\left|a_{2}\right|<1$ exactly states that $r_{1}+r_{2}<d$, ie the closed discs $D\left(x_{i}, r_{i}\right), i=1,2$, are disjoint. Fix $\varepsilon>0$ so small that $r_{1}+r_{2}+2 \varepsilon<d$, and set $\rho_{i}=r_{i}+\varepsilon$.

Obviously, the image of $D_{i}=D\left(x_{i}, \rho_{i}\right)$ by $A_{i}$ is contained in the interior of $D_{i}$. Now let us look at the image of $D_{2}$ by $A_{1}$. Take $x \in D_{2}$. Then

$$
\begin{aligned}
\left|A_{1} x-x_{1}\right| & \leq\left|A_{1} x-A_{1} x_{2}\right|+\left|A_{1} x_{2}-x_{1}\right| \\
& \leq\left|a_{1}\right|\left|x-x_{2}\right|+\left|A_{1} x_{2}-A_{1} x_{1}\right| \\
& \leq\left|a_{1}\right|\left(r_{2}+\varepsilon\right)+\left|a_{1}\right| d=r_{1}+\left|a_{1}\right| \varepsilon<\rho_{1} .
\end{aligned}
$$

A similar inequality is obtained by permuting 1 and 2 .

Thus $A_{i}\left(D_{1} \cup D_{2}\right)$ is contained in the interior of $D_{i}$. Let now $B_{i}$ denote the closed half space defined by $D_{i}$. Then, multiplying the metric on $\mathbf{H}_{\mathbf{R}}^{3}$ by a suitable constant, $B_{1}$ and $B_{2}$ satisfy all the hypotheses of Lemma 2.1.

The ultrametric case is analogous and even simpler. Consider the attractive point $x_{1}$ of $A_{i}$ in $\mathbf{P}^{1}(\mathbf{K})$, and set $d=d\left(x_{1}, x_{2}\right)$. Take for $D_{i}$ the open ball of centre $x_{i}$ and radius $d$. This is actually a closed ball of strictly smaller radius (because the metric takes a discrete set of values) and $D_{1} \cap D_{2}=\varnothing$ (because $d$ is an ultrametric). Now $D_{1}$ and $D_{2}$ are the boundaries of two disjoint half-trees $B_{1}$ and $B_{2}$ in the tree of $\mathrm{PGL}_{2}(\mathbf{K})$, satisfying all the hypotheses of Lemma 2.1.

Remark 3.2 In the ultrametric case, it is not hard to show that the sufficient condition $\max \left(\left|a_{1}\right|,\left|a_{2}\right|\right)<1$ is necessary so as to have a quasi-isometrically embedded free semigroup.

The Archimedean case is more involved. When $\mathbf{K}=\mathbf{R}$, the condition $\left|a_{1}\right|+\left|a_{2}\right|+$ $3\left|a_{1}\right|\left|a_{2}\right|<1$ is not optimal and can be replaced by the weaker condition $\left|a_{1}\right|+\left|a_{2}\right|<1$; this can be shown directly by applying the quasi-isometric ping-pong Lemma to suitable intervals that are not centered at the attractive points; moreover the usual ping-pong Lemma applies when $\left|a_{1}\right|+\left|a_{2}\right| \leq 1$ (always supposing $\max \left(\left|a_{1}\right|,\left|a_{2}\right|\right)<1$ ) but the free semigroup can be non-quasi-isometrically embedded. For instance, if

$$
A_{1}=\left(\begin{array}{cc}
1 / 2 & 0 \\
0 & 1
\end{array}\right) \text { and } A_{2}=\left(\begin{array}{cc}
1 / 2 & 1 \\
0 & 1
\end{array}\right)
$$


then $A_{1}$ and $A_{2}$ generate a free semigroup, but a direct calculation shows that $A_{1} A_{2}^{n-1}$ and $A_{2} A_{1}^{n-1}$ remain at bounded distance.

Finally, the complex case seems even more tricky, as for instance there is no simple characterization of the elements $a \in \mathbf{C}$ such that the matrices

$$
A_{1}=\left(\begin{array}{ll}
a & 0 \\
0 & 1
\end{array}\right) \text { and } A_{2}=\left(\begin{array}{ll}
a & 1 \\
0 & 1
\end{array}\right)
$$

generate a quasi-isometrically embedded semigroup; it easily implies $|a|<1$ and can be shown to be true if $|a|<1 / 2$, but it is also true for some other values of modulus between $1 / 2$ and 1 .

\section{Free sub-semigroups in Lie groups and $p$-adic groups}

We make use of the following unpublished lemma of Guivarc'h.

Recall that a finite-dimensional Lie algebra over $\mathbf{R}$ is called of type $\mathbf{R}$ if for every $x$ the eigenvalues of $\operatorname{ad}(x)$ are in $i \mathbf{R}$, and a connected Lie group is called of type $\mathbf{R}$ if its Lie algebra is of type R.

Lemma 4.1 Let $G$ be a connected solvable real Lie group. Suppose that $G$ is not of type $R$. Then $G$ has a quotient $Q$ isomorphic to a closed (in the ordinary topology) subgroup of the affine complex group $\mathbf{C}^{*} \ltimes \mathbf{C}$ (which is also isomorphic to the group of orientation-preserving similarities of $\mathbf{R}^{2}$ ). More precisely, $Q$ is isomorphic to one of the following groups

- $\mathbf{R}_{+}^{*} \ltimes \mathbf{R}$;

- $\mathbf{C}^{*} \ltimes \mathbf{C}$;

- $\mathbf{R}_{+}^{*} \ltimes \mathbf{C}$, with $\mathbf{R}_{+}^{*}$ acting by a one parameter subgroup of similarities that is neither an action by homotheties, nor an action by rotations.

We make use of the following standard lemma.

Lemma 4.2 Let $\mathfrak{g}$ be a real solvable finite-dimensional Lie algebra over $\mathbf{R}$, and let $\mathfrak{n}$ be its nilpotent radical. Suppose that $\mathfrak{g} /[\mathfrak{n}, \mathfrak{n}]$ is of type $R$. Then $\mathfrak{g}$ is of type $R$.

Proof Denote by $\mathfrak{n}$ the nilpotent radical of $\mathfrak{g}$, and $\mathfrak{n}_{\mathbf{C}}$ its complexification. It can be decomposed as a sum of characteristic subspaces under the adjoint action, denoted $\mathfrak{n}_{\alpha}$ for $\alpha \in \operatorname{Hom}(\mathfrak{g}, \mathbf{C})$. We have $\left[\mathfrak{n}_{\alpha}, \mathfrak{n}_{\beta}\right]=\mathfrak{n}_{\alpha+\beta}$ for all $\alpha, \beta$. Taking a similar decomposition for the action on $\mathfrak{n} /[\mathfrak{n}, \mathfrak{n}]$, we see that if $\mathfrak{g} /[\mathfrak{n}, \mathfrak{n}]$ is of type $R$, then $\mathfrak{g}$ is also of type $\mathrm{R}$ (ie $\mathfrak{n}_{\alpha} \neq 0$ only if $\alpha \in i \operatorname{Hom}(\mathfrak{g}, \mathbf{R})$ ). 
Proof of Lemma 4.1 Let $\mathfrak{g}_{0}$ be the Lie algebra of $G$. Then $\mathfrak{g}_{0}$ has a quotient $\mathfrak{g}$ that is not of type $\mathrm{R}$, but all of whose proper quotients are of type $\mathrm{R}$. Let $\mathfrak{n}$ be the nilpotent radical of $\mathfrak{g}$. It follows from Lemma 4.2 that $\mathfrak{n}$ is abelian.

By Bourbaki [3, Chapter 7], there exists a Cartan subalgebra, that is a nilpotent subalgebra $\mathfrak{h}$ of $\mathfrak{g}$ such that $\mathfrak{g}=\mathfrak{h}+\mathfrak{n}$. Let $\mathfrak{z}$ be the centre of $\mathfrak{h}$. Then $\mathfrak{z} \cap \mathfrak{n}$ centralizes both $\mathfrak{h}$ and $\mathfrak{n}$, hence is central in $\mathfrak{g}$. As being of type $\mathrm{R}$ is preserved by central extensions, $\mathfrak{g} /(\mathfrak{z} \cap \mathfrak{n})$ is not of type $R$, so that $\mathfrak{z} \cap \mathfrak{n}=0$. Therefore $\mathfrak{h} \cap \mathfrak{n}$ is an ideal in $\mathfrak{h}$ having trivial intersection with the centre of $\mathfrak{h}$; as $\mathfrak{h}$ is nilpotent, this implies that $\mathfrak{h} \cap \mathfrak{n}=0$, so that $\mathfrak{g} \simeq \mathfrak{n} \rtimes \mathfrak{h}$.

So $\mathfrak{h} \simeq \mathfrak{g} / \mathfrak{n}$ is abelian, and $\mathfrak{n}$ is an irreducible $\mathfrak{h}$-module; in particular it has dimension at most 2. Moreover as $\mathfrak{n}$ is the nilpotent radical, the action of $\mathfrak{h}$ is faithful. This also implies that $\mathfrak{h}$ has dimension at most 2 . Looking more closely at irreducible abelian subalgebras in $\mathfrak{g l}_{n}(\mathbf{R})$ for $n=1,2$, we get the desired list.

This proves that if $\widetilde{G}$ is the universal covering of $G$, then it has a closed connected normal subgroup $W$ such that $L=\widetilde{G} / W$ is isomorphic to one of the groups given in the lemma. Now the image of the centre of $\widetilde{G}$ maps to the centre of $L$, which is trivial in all cases. This means that the centre of $\widetilde{G}$ is contained in $W$, and in particular $L$ is a quotient of $G$.

Theorem 4.3 Let $G$ be a connected amenable Lie group with non-polynomial growth. Then $G$ has a quasi-isometrically embedded free sub-semigroup on 2 generators.

Proof A connected amenable Lie group has cocompact radical. As the embedding of a cocompact subgroup is obviously quasi-isometric, we can suppose $G$ solvable. By Lemma 4.1, $G$ has a quotient which is a closed subgroup of $\mathbf{C}^{*} \ltimes \mathbf{C}$. As we can lift a quasi-isometrically embedded semigroup from any quotient, we can suppose that $G$ is a closed subgroup of $\mathbf{C}^{*} \ltimes \mathbf{C}$ given as in Lemma 4.1. Now $\mathbf{C}^{*} \ltimes \mathbf{C}$ is the stabilizer of the point at infinity $\infty \in \mathbf{P}^{1}(\mathbf{C})$, for the isometric action of $\mathrm{PGL}_{2}(\mathbf{C})$ on the hyperbolic 3 -space $\mathbf{H}_{\mathbf{R}}^{3}$. Hyperbolic elements inside $\mathbf{C}^{*} \ltimes \mathbf{C}$ are elements $(a, b)$ with $|a| \neq 1$, they have origin $\infty$ if and only if $|a|<1$. Two such hyperbolic elements have the same target if and only if they commute. We thus immediately see that in all cases given by Lemma 4.1, there are two hyperbolic elements with origin $x_{0}$ and distinct targets; taking suitable powers we get by Lemma 3.1 two generators of a quasi-isometrically embedded semigroup.

Let $\mathbf{K}$ be a local field of characteristic zero, and $G$ a solvable connected linear algebraic $\mathbf{K}$-group. Decompose it as $G=D K U$, where $U$ is the unipotent radical, $D$ is a maximal split torus, and $K$ is compact. We say that $G$ is of type $\mathrm{R}$ if $[D, U]=1$. This 
is equivalent to say that $K U$ (which does not depend on the choice of the Levi factor) is almost a direct factor in $G$. In this case $G$ has a cocompact nilpotent subgroup, namely $D U$.

Lemma 4.4 Let $\mathbf{K}$ be a local field of characteristic zero, and $G$ a solvable linear algebraic $\mathbf{K}$-group that is not of type $R$. Then $G$ has a cocompact subgroup (namely, $D U$ as above) having the affine group $\mathbf{K}^{*} \ltimes \mathbf{K}$ as a quotient.

Proof We can diagonalize the action of $D$ on the Lie algebra $\mathfrak{u}$ of $U$, writing $\mathfrak{u}=$ $\bigoplus \mathfrak{u}_{\alpha}$, where $\alpha \in \operatorname{Hom}\left(D, \mathbf{K}^{*}\right) \simeq \mathbf{Z}^{n}(n$ being the rank of $D)$. We have $\left[\mathfrak{u}_{\alpha}, \mathfrak{u}_{\beta}\right]=\mathfrak{u}_{\alpha \beta}$ for all $\alpha, \beta$. It follows that $\mathfrak{u}_{1}$ is a Lie subalgebra. By assumption $\mathfrak{u}_{1} \neq \mathfrak{u}$. Therefore, as $\mathfrak{u}$ is nilpotent, we have $\mathfrak{u}_{1}+[\mathfrak{u}, \mathfrak{u}] \neq \mathfrak{u}$. It follows that $G /[U, U]$ is not of type $\mathrm{R}$, so that we are reduced to the case when $U$ is abelian. As the action of $D$ on $U$ is diagonalizable, taking again a quotient if necessary we can suppose that $U$ is one-dimensional and that $D$ acts faithfully on $U$. In this latter case, $G$ is isomorphic to the affine group.

Theorem 4.5 Let $G$ be a linear algebraic group over a field of characteristic zero. Suppose that $G$ is compactly generated of non-polynomial growth. Then $G$ has a quasi-isometrically embedded free sub-semigroup on 2 generators.

The proof, which makes use of Lemma 4.4, is similar to that of Theorem 4.3 and we omit it.

\section{Free sub-semigroups in finitely generated solvable groups}

Theorem 5.1 Let $\Gamma$ be a finitely generated solvable, non-virtually-nilpotent group. Then $\Gamma$ has a quasi-isometrically embedded free sub-semigroup on 2 generators.

Proof By a result of Groves [8], taking a finite subgroup of finite index if necessary there exists an infinite field $K$ and a homomorphism $\Gamma \rightarrow K^{*} \ltimes K$ with Zariski dense image.

Then the image contains two non-commuting elements $A_{1}=\left(a_{1}, b_{1}\right)$ and $A_{2}^{\prime}=\left(a_{2}^{\prime}, b_{2}^{\prime}\right)$ where $a_{1}, a_{2}$ are not roots of unity. Indeed, the image of $\Gamma$ in $K^{*}$ is finitely generated, and therefore its torsion subgroup $T$ is finite. Then the preimage in $K^{*} \ltimes K$ of $K^{*}-T$ is an open Zariski dense subset, so that its intersection with $\Gamma$ is also Zariski dense, and therefore contains two non-commuting elements.

By a lemma of Tits [15], there exists a local field $\mathbf{K}$ and an embedding of $K$ in $\mathbf{K}$ so that $\left|a_{1}\right|<1$ in $\mathbf{K}$. Then for sufficiently large $n \geq 0$, we have, setting $A_{2}=A_{2}^{\prime} A_{1}^{n}$ 
and $A_{2}=\left(a_{2}, b_{2}\right)$, the inequality $a_{1}+a_{2}+3 a_{1} a_{2}<1$. Therefore by Lemma 3.1, the elements $A_{1}$ and $A_{2}$ generate a quasi-isometrically embedded free semigroup in $\mathbf{K}^{*} \ltimes \mathbf{K}$. Therefore if we lift them in $\Gamma$, we get a quasi-isometrically embedded free semigroup.

The following (classic) remark shows that all cases above ( $\mathbf{K}$ of all characteristics, and, in characteristic zero, both ultrametric and Archimedean cases) were necessary to consider in the proof of Theorem 5.1.

\section{Remark 5.2}

(1) Let $P$ be the polynomial $X^{2}-\frac{6}{5} X+1$. This is the minimal polynomial over $\mathbf{Q}$ of the element $(3+4 i) / 5$ of $\mathbf{C}$ (which has modulus 1 ). Set $G=\mathbf{Z} \ltimes \mathbf{Z}[1 / 5]^{2}$, where $\mathbf{Z}$ acts by the companion matrix of $P$. Then $G$ is a finitely generated metabelian group with exponential growth, but for every representation of $G$ in $\mathrm{GL}_{n}(\mathbf{R})$ for any $n$, the Zariski closure of the image has polynomial growth.

(2) Let $Q$ be the polynomial $X^{3}+X+1$. It is straightforward that if $\alpha$ is a root of $Q$ in an ultrametric local field, then $|\alpha|=1$. Set $G=\mathbf{Z} \ltimes \mathbf{Z}^{3}$, where $\mathbf{Z}$ acts by the companion matrix of $Q$. Then $G$ is a polycyclic metabelian group with exponential growth, but for every representation of $G$ in an ultrametric local field, the closure of the image has polynomial growth.

(3) If $p$ is prime and $G=(\mathbf{Z} / p \mathbf{Z}) \prec \mathbf{Z}$ (wreath product), any linear representation of $G$ in characteristic $\neq p$ has virtually abelian image.

(4) If $G$ is polycyclic, or is the Baumslag-Solitar group $\mathbf{Z} \ltimes_{n} \mathbf{Z}[1 / n]$, or more generally if $G$ is solvable and virtually torsion-free, then any linear representation of $G$ in characteristic $p>0$ has virtually abelian image.

\section{References}

[1] S I Adian, Random walks on free periodic groups, Math. USSR Izvestiya 21 (1983) 425-434

[2] I Benjamini, O Schramm, Every graph with a positive Cheeger constant contains a tree with a positive Cheeger constant, Geom. Funct. Anal. 7 (1997) 403-419 MR1466332

[3] N Bourbaki, Groupes et algèbres de Lie. Chapitres 4, 5 et 6, Éléments de mathématique, Masson, Paris (1981) MR647314

[4] J Bourgain, The metrical interpretation of superreflexivity in Banach spaces, Israel J. Math. 56 (1986) 222-230 MR880292 
[5] E Breuillard, On uniform exponential growth for solvable groups, Pure and Applied Math Quarterly 3(4) (2007) 949-967 Special issue: in honor of G. Margulis, Part I

[6] B Farb, The extrinsic geometry of subgroups and the generalized word problem, Proc. London Math. Soc. (3) 68 (1994) 577-593 MR1262309

[7] M Gromov, Asymptotic invariants of infinite groups, from: "Geometric group theory, Vol. 2 (Sussex, 1991)", London Math. Soc. Lecture Note Ser. 182, Cambridge Univ. Press, Cambridge (1993) 1-295 MR1253544

[8] J R J Groves, Soluble groups with every proper quotient polycyclic, Illinois J. Math. 22 (1978) 90-95 MR474887

[9] A Y Ol'shanskir,, On the distortion of subgroups of finitely presented groups, Mat. Sb. 188 (1997) 51-98 MR1601512

[10] D Osin, Small cancellations over relatively hyperbolic groups and embedding theorems arXiv:math.GR/0411039

[11] D Osin, Subgroup distortions in nilpotent groups, Comm. Algebra 29 (2001) 5439-5463 MR1872804

[12] J M Rosenblatt, Invariant measures and growth conditions, Trans. Amer. Math. Soc. 193 (1974) 33-53 MR0342955

[13] R Tessera, Asymptotic isoperimetry on groups and uniform embeddings into Banach spaces arXiv:math.GR/0603138

[14] R Tessera, Large-scale Sobolev inequalities on metric measure spaces arXiv: math.MG/0702751

[15] J Tits, Free subgroups in linear groups, J. Algebra 20 (1972) 250-270 MR0286898

IRMAR, Campus de Beaulieu, 35042 Rennes Cedex, France

Department of Mathematics, Stevenson Center, Vanderbilt University

Nashville TN 37240, USA

yves.decornulier@univ-rennes1.fr, romain.a.tessera@vanderbilt.edu

Proposed: Martin Bridson

Seconded: Walter Neumann. Jean-Pierre Otal
Received: 15 December 2006

Revised: 19 December 2007 